\title{
Sample preparation procedures for analysis of organochlorinated pollutants and PAHs in surface water samples
}

\author{
Olga Vyviurska, Ivan Špánik \\ Slovak University of Technology, Institute of Analytical Chemistry, \\ Radlinského 9, SK-81237 Bratislava, Slovak Republic \\ ivan.spanik@stuba.sk
}

\begin{abstract}
This review focuses on sample preparation procedures used for chromatographic analysis of the most common organochlorinated pollutants and PAHs in water samples. The studied pollutants have a long persistence in environment, possible carcinogenic effects, and their application has been banned in many countries. The standard ISO procedures with the modern sample preparation procedures were compared in the term of recovery effectiveness for target analytes.
\end{abstract}

Keywords: sample preparation procedures, chromatography, organochlorinated pollutants, PAHs

\section{Introduction}

Sample preparation procedure is a crucial step of trace analysis, which mainly defines the accuracy of the obtained results. Nowadays, traditional sample preparation procedures used for chromatography, e.g. liquid-liquid extraction (LLE) and solid phase extraction (SPE) are slowly replaced by their miniaturized versions. For example, a thin film of sorbent could be coated on a fused-silica fibre (SPME) or a stir bar (SBSE). As to liquid microextraction procedures, the extraction of pollutants is successfully performed on a microdrop of organic solvent suspended from the tip of a microsyringe needle in the case of single-drop microextraction. At the same time, drops of the mixture of a scattering solvent with extraction solvent are used for dispersive liquid-liquid microextraction. Furthermore, separation procedure could be also occurred though flat-sheet and hollow-fibre membrane, where separation mechanisms varies depending on the membrane nature. Size-exclusion mechanism is typical for porous membrane (e.g. polypropylene glycol), both size and charge differences define ionexchange membrane effectiveness, and partition coefficients are specific parameters for non-porous membrane composed of polymeric film. In addition, membrane pores could be impregnated with a water-immiscible organic solvent to enhance the separation capability. Application of these new sample preparation techniques yields to a significantly reduced consumption of toxic organic solvents, decreased sample amount and improvement of detection limits.

Organochlorinated pollutants and polycyclic aromatic hydrocarbons (PAHs) belong to the most common priority substances defined the European
Water Framework Directive, which mandatory limits of detection in range of $\mathrm{ng} / \mathrm{l}$. Organochlorinated pollutants are represented by agricultural insecticides and polychlorinated biphenyls (PCBs). Some of insecticides are persistent in environment due to their problematic degradation by microorganisms, and as a result, their utilization is restricted in many countries. In addition, organochlorinated pesticides are commonly included by International Agency for Research of Cancer (IARC) in Group 2B "possibly carcinogenic to humans", as well as in Toxic Substances and Disease Registry (U.S. Agency), because of its effects on endocrine, neurological and reproductive human organ systems. One of the first insecticides, dichlorodiphenyltrichloroethane (DDT) have been synthesized in form of two isomers, but only $p, p$-DDT was used commercially. Dichlorodiphenyldichloroethylene (DDE) and dichlorodiphenyldichloroethane (DDD) are degradation products of DDT, and usually found as contaminants of DDT preparation process. Hexachlorocyclohexanes (HCH) represents the group of 9 isomers, where the special attention should be paid on its $\gamma$-form (lindane), whereas the other forms are mostly the byproducts of lindane synthesis. Aldrin is easily converted into dieldrin in environment and living organisms, and characterized by low mobility in soil and advanced bioaccumulation properties. Heptachlor is usually found in stable form of heptachlor epoxide in environmental samples, because of the bacterial influence. In common, endosulfan is not detected in waters, only in agricultural runoff and rivers in industrial zones and hazardous waste localities.

Polychlorinated biphenyls represent a nonpolar organic hydrocarbons group of 209 congeners containing from one to ten chlorine atoms connected 
to a pair of linked benzene rings. In the US the mixture of PCBs has the technical name Aroclor with 4 numbers specification, where the first two figures point on the number of carbon atoms in the phenyl rings and the second two indicate the percentage of chlorine by mass in the mixture, e.g. Aroclor 1242, Aroclor 1254 and Aroclor 1260. In other countries PCBs commercial mixtures were marked under other names, e.g. Clophen (Germany), Kanechlor (Japan), Delor (Czechoslovakia) and Sovol (USSR). Coplanar configuration of PCBs is found for those congeners with chlorine substitution at the para and at least two meta positions on biphenyl nucleus, but for non-ortho substitutions. It was reported about high individual toxicities of such PCBs (PCBs $77,81,126,169)$, but usually these congeners are rarely present in environmental samples (at level of the parts of trillion range). A single ortho-chloro substituted PCBs (105, 114, 118, 123, 156, 157, $167,189)$ are also relatively coplanar and consequently demonstrate enzyme inducing potencies (McFarland et al. 1989). These non-ortho-coplanar and mono-ortho-chloro congeners are also called "dioxin-like" or "WHO" PCBs. The toxicity of particular PCB depends on similarity of its molecular spatial configuration to molecular structure 2,3,7,8TCDD (2,3,7,8-tetrachlorodibenzo- $p$-dioxin). Some di-ortho coplanar PCB molecules (128, 138, 158, $166,168,170)$ are also potential toxicants, especially congeners 138 and 153 which are the major components of PCB technical mixtures. In common, enviromental samples are usually analyzed for their content of the seven European indicator PCB (28, 52, 101, 118, 138, 153, 180) and the 36 McFarland and Clarke PCBs $(18,37,44,49,52,70,74,77,81$, $87,99,101,105,114,118,119,123,126,128,138$, $151,153,156,157,158,167,168,169,170,177,180$, 183, 187, 189, 194, 201) (Larsen 1995).

PAHs as products of incomplete combustion of organic compounds are supplied into the environment in the form of resins, oils, coal, automobile exhaust emissions and cigarette smoke. PAHs show high human carcinogenic and mutagenic effect inducing oxidative stress and oxidative DNA damage, and are easily concentrated through biological chain (Ma et al. 2010). The following 16 PAHs are required by the United States Environmental Protection Agency (US-EPA) to control in water: acenaphthene, acenaphthylene, anthracene, benzo $[a]$ anthracene, benzo $[a]$ pyrene, benzo $[b]$ fluoranthene, benzo[ $g, h, i]$ perylene, benzo[k] fluoranthene, chrysene, dibenzo $[a, h]$ anthracene, fluoranthene, fluorine, indeno[1,2,3-c,d]pyrene, naphthalene, phenanthrene, and pyrene, and 8 of them are also recommended by the EU legislation.

\section{Sample preparation procedures for organochlorinated pollutants}

The ISO 6468:1996 procedure proposed liquid-liquid extraction with hexane, heptane or petrolether as sample preparation procedure for extraction of organochlorine insectecides and polychlorinated biphenyls from surface waters. Previously the acidity of the water samples $(30-100 \mathrm{ml})$ should be regulated to $\mathrm{pH} 5-7.5$ value, whereas for water sample with endosulfane to $\mathrm{pH} 2$. Derouiche et al. tested two commercial solid phase microextraction (SPME) fibres with different thickness of coating such as PDMS (7, 30, $100 \mu \mathrm{m}$ film thicknesses) and PDMS-DVB (65 $\mu \mathrm{m}$ film thickness) for determination of 15 OCPs and 20 PCBs in aqueous samples (Derouiche et al. 2007). PDMS-DVB fibre was more effective for extraction of high volatile compounds (especially di-, tri- and tetra-PCBs), whereas other target analytes were more effectively concentrated with PDMS fibre $(100 \mu \mathrm{m})$ under the selected experimental conditions (Table 1). Camino-Sánchez et al. validated a stir bar sorptive extraction (SBSE) method for the simultaneous analysis of 77 POPs including 10 PCBs in river water (Camino-Sánchez et al. 2012). Preconcentration procedure was carried out from $200 \mathrm{ml}$ of water with $10 \mathrm{~g}$ of $\mathrm{NaCl}$ by PDMS stir bar (0.5 mm thickness, $20 \mathrm{~mm}$ lenght) for $24 \mathrm{~h}$. It should be noticed that this method provided a possibility to analyze a large number of analytes in one run with very low LOQ values. RSD values increased with increasing number of chlorine atoms in PCB structure, and quantification characteristics of method were sufficient for analysis of such complicated mixture.

In spite of SPME, a micro-drop of organic solvent suspended from the tip of a microsyringe which is used for single drop microextraction (SDME) procedure could be renewed for each experiment. The organochlorine pesticides were successfully extracted by $1.8-2 \mu \mathrm{l}$ of toluene from $5-10 \mathrm{ml} \mathrm{sam}$ ple during 15-37 min at constant stirring (Lambropoulou et al. 2007; Cortada et al. 2009a). Although the recovery characteristics of used SDME was high for most of studied compounds, the sensitivity of the procedure was lower than for mentioned above SBSE and SPME techniques. Polypropylene hollow fiber $(600 \mu \mathrm{m}$ inner diameter, $200 \mu \mathrm{m}$ thickness of the wall, $0.2 \mu \mathrm{m}$ pore size) filled with $10-20 \mu \mathrm{L}$ toluene as acceptor phase was applied for hollowfiber liquid-phase microextraction (HF-LPME) for concentration of organochlorine insecticides from $1.8-5 \mathrm{ml}$ of water sample. As the result the obtained LoD values were lower than for SDME (Lambropoulou et al. 2007).

In the case of dynamic hook-type liquid-phase microextraction (DHT-LPME), a polypropylene 
hollow fibre with hook shape inserted into a microsyringe filled with $35 \mu$ l of 1-hexanol was placed in the headspace above $10 \mathrm{ml}$ of water sample. The extraction occurs on a large drop (13-14 $\mu$ l) formed inside the fibre. The LoD value (2-5 ng/L) for the proposed analytical procedure was near to these required by ISO 6468:1996 technique (Chen et al. 2009). The modified version of DHT-LPME, dynamic headspace time-extended helix liqiud-phase microextraction (DHS-TEHLPME) procedure utilized the microsyringe with $13 \mu$ portion of 1 -octanol revolved around with hollow fibre with 1-octanol impregnated pores (Huang et al. 2009). Such equipment was applied for headspace preconcentration from a $110 \mathrm{ml}$ aqueous sample. Since $10 \mu \mathrm{l}$ of the received extract should be injected, special inlet is required. The promising results were received for $o, p$-DDT and aldrin, for which observed LoD was lower than $1 \mathrm{ng} / \mathrm{L}$. Dispersive liquid-liquid mictroextraction (DLLME) with $10 \mu \mathrm{L}$ of tetrachloroethylene (extraction solvent) and $1 \mathrm{~mL}$ of acetone (disperser solvent) was used for extraction procedure of $10 \mathrm{ml}$ of water sample (Cortada et al. 2009b). After centrifugation procedure of cloudy solution, $2 \mu \mathrm{L}$ of extractant was recovered and injected into the GC-MS instrument. This procedure provided much improved sensitivity for organochlorine insectecides compared to SDME. In spite of HF-LPME, membrane-assisted extraction (MASE) utilizes non-porous membrane since porous membrane has as memory effects (Lambropoulou et al. 2007). Also up to $1 \mathrm{ml}$ of solvent could be immobilized in this case, whereas only 10-20 $\mu$ l of solvent in HF-LPME. The membrane is commonly inexpensive and could work as a filter to prevent extraction of larger molecules and interferences for sample clean-up procedures (Lambropoulou et al. 2007) e.g. flat membrane from low-density polyethylene glycol (Hauser et al. 2001), dense polypropylene bags (Hauser et al. 2004). Nonpolar solvents (heptane, cyclohexane) were preferred as acceptor phase. It should be noticed that in comparison with the ISO procedure, better LoDs was received with MASE for most of target compounds. The main disadvantages of MASE procedure were long extraction time (12 h) and its limitation for polar compounds, because of the nonpolar character of the used membrane. In dispersive liquid-liquid microextraction with solidification of a floating organic drop (DLLMESFO), a single microdroplet $(2 \mu \mathrm{L})$ or a few droplets (total volume $10 \mu \mathrm{L}$ ) could be used for extraction (Yamini et al. 2008; Leong et al. 2009). Nonpolar hexadecane or polar 1-dodecanol organic solvents were applied for 5-20 $\mathrm{ml}$ of water sample. In both case, the sample was cooled by inserting into an ice bath, and $1-2 \mu \mathrm{L}$ of extract was injected. Similarly to DLLME-SFO, small amounts of solvent was required $(13 \mu \mathrm{L}$ of mixed organic solvent (TCE:TBME $=4: 6$ ) in dispersive liquid-liquid microextraction with little solvent consumptions (DLLE-LSC) for $10 \mathrm{ml}$ of sample (Tsai et al. 2009). High recoveries were received in this case (90.5-109.4\%). Ultrasonic radiation was efficient for acceleration of mass transfer process between two immiscible phases (Ozcan et al. 2009; Regueiro et al. 2008). Extraction with chloroform $(100-200 \mu \mathrm{l})$ was performed from $10 \mathrm{ml}$ of water sample in an ultrasonic water bath for 5-10 $\mathrm{min}$. In general, LoDs of the determined compounds were higher than the recommended values by ISO procedure, but the improved recoveries (83-103\%) were obtained with DLLME-SFO procedure.

As to PCBS the PDMS fibre (30 $\mu \mathrm{m}$ film coating) was used for concentration of the congeners from water sample in presence of PAHs and phthalate esters (Cortazar et al. 2002). In this case, a larger amount of water sample was required for extraction, but much worse LoD values were received compared to the other similar investigations. Polydimethylsiloxane (PDMS, $100 \mu \mathrm{m}$ ) SPME fiber and porous polypropylene hollow fibre (PC-HFME) with 2,5-substitued pyridine were compared for the extraction of $\mathrm{PCB}$ congeners from a $10 \mathrm{ml}$ coastal sea water sample (Basheer et al. 2007). Although the developed method of PG-HFME demanded utilization of desorption procedure with $100 \mu \mathrm{l}$ of hexane and ultrasonication, it was highly reproducible with better extraction efficiency than SPME. A single drop of immiscible solvent suspended in a sample (SDME) and extraction through a membrane (MASE) were applied for concentration of PCB congeners from environmental samples (Table 1) (Lambropoulou et al. 2007). $5 \mathrm{ml}$ of sample was extracted with a $1.6 \mu \mathrm{l}$ drop of toluene during $15 \mathrm{~min}$ in the case of SDME, and whereas $15 \mathrm{ml}$ of the $\mathrm{NaCl}$ saturated aqueous sample was concentrated into $800 \mu \mathrm{l}$ of cyclohexane though polypropylene membrane during $30 \mathrm{~min}$ at constant shaking in MASE. In this case, SDME preconcentration procedure was more effective as to the received LoD and recovery values. Razaei et al. (Rezaei et al. 2008) tried DLLME with $500 \mu \mathrm{L}$ acetone as dispersive solvent and $10.0 \mu \mathrm{L}$ of chlorobenzene as extraction solvent for $5 \mathrm{ml}$ of PCBs spiked water sample. LoD values of this procedure was improved compared to previously described SPME (Cortazar et al. 2002), SDME (Lambropoulou et al. 2007) and MASE (McFarland et al.1989) sample preconcentration techniques. 
Tab. 1. Sampe preparation procedure for organochlorine insectecides.

\begin{tabular}{|c|c|c|c|c|c|c|c|}
\hline $\begin{array}{l}\text { Sample } \\
\text { preparation }\end{array}$ & Compounds & Type of water & Analysis & $\begin{array}{l}\text { LoD } \\
(\mathbf{n g} / \mathbf{l})\end{array}$ & $\begin{array}{l}\text { Recovery } \\
(\%)\end{array}$ & RSD (\%) & Ref. \\
\hline \multirow[t]{12}{*}{ SPME } & aldrin & River water & GC-MS & 4.5 & 96.2 & 11.34 & $\begin{array}{l}\text { Derouiche et al. } \\
2007\end{array}$ \\
\hline & dieldrin & River water & GC-MS & 8.1 & 91.5 & 15.86 & \\
\hline & $p, p^{\prime}$-DDT & River water & GC-MS & 26.3 & 76.3 & 19.7 & \\
\hline & $o, p^{\prime}$-DDE & River water & GC-MS & 0.4 & 83.6 & 16.45 & \\
\hline & $p, p-\mathrm{DDE}$ & River water & GC-MS & 1.0 & 89.7 & 16.45 & \\
\hline & $o, p^{\prime}$-DDD & River water & GC-MS & 7.2 & 87.2 & 20.64 & \\
\hline & $\gamma-\mathrm{HCH}$ & River water & GC-MS & 1.6 & 101.2 & 11.64 & \\
\hline & hexachlorobenzene & River water & GC-MS & 16.2 & 105.1 & 13.94 & \\
\hline & heptachlor & River water & GC-MS & 22.0 & 105.2 & 11.79 & \\
\hline & heptachlor epoxide & River water & GC-MS & 4.3 & 96.3 & 6.12 & \\
\hline & PCBs & $\begin{array}{l}\text { An artificial sea water, } \\
\text { spiked tap water }\end{array}$ & GC-MS & $0.13-4.63$ & $83-93$ & $4.4-15.7$ & Basheer et al. 2007 \\
\hline & & Water & GC-MS & $30-110$ & & $8-14$ & Cortazar et al. 2002 \\
\hline \multirow[t]{16}{*}{ SBSE } & aldrin & River water & GC-MS & $0.25^{*}$ & 111 & 8 & $\begin{array}{l}\text { Camino-Sánchez } \\
\text { et al. } 2012\end{array}$ \\
\hline & dieldrin & River water & GC-MS & $0.25^{*}$ & 100 & 16 & \\
\hline & endrin & River water & GC-MS & $1.50 *$ & 92 & 14 & \\
\hline & $p, p^{2}$-DDT & River water & GC-MS & $0.25^{*}$ & 101 & 10 & \\
\hline & $p, p^{2}-\mathrm{DDT}+p, p^{2}-\mathrm{DDD}$ & River water & GC-MS & $0.50 *$ & 97 & 14 & \\
\hline & $o, p^{\prime}$-DDT & River water & GC-MS & $0.25^{*}$ & 103 & 15 & \\
\hline & $\alpha$-endosulfan & River water & GC-MS & $0.25^{*}$ & 100 & 13 & \\
\hline & $\beta$-endosulfan & River water & GC-MS & $1.50^{*}$ & 96 & 16 & \\
\hline & $\alpha-\mathrm{HCH}$ & River water & GC-MS & $0.25^{*}$ & 98 & 14 & \\
\hline & $\beta-\mathrm{HCH}$ & River water & GC-MS & $1.50 *$ & 99 & 12 & \\
\hline & $\delta-\mathrm{HCH}$ & River water & GC-MS & $1.50 *$ & 100 & 16 & \\
\hline & heptachlor & River water & GC-MS & $0.25^{*}$ & 100 & 10 & \\
\hline & heptachlor epoxide & River water & GC-MS & $0.25 *$ & 98 & 12 & \\
\hline & pentachlorobenzene & River water & GC-MS & $0.25 *$ & 101 & 24 & \\
\hline & hexachlorobenzene & River water & GC-MS & $2.50 *$ & 105 & 11 & \\
\hline & PCBs & River water & GC-MS & $10 *$ & $76-105$ & $6-24$ & $\begin{array}{l}\text { Camino-Sánchez } \\
\text { et al. } 2012\end{array}$ \\
\hline \multirow[t]{14}{*}{ SDME } & aldrin & Water, wastewater & GC-MS & 53 & $90 \pm 8$ & 9.9 & Cortada et al. 2009a \\
\hline & dieldrin & & GC-ECD & 5 & $96.7-96.1$ & 5.7 & $\begin{array}{l}\text { Lambropoulou } \\
\text { et al. } 2007\end{array}$ \\
\hline & dieldrin & Water, wastewater & GC-MS & 53 & $90 \pm 8$ & 9.9 & \\
\hline & dieldrin & Water, wastewater & GC-MS & 22 & $78 \pm 8$ & 6.3 & Cortada et al. 2009a \\
\hline & endrin & Water, wastewater & GC-MS & 68 & $78 \pm 6$ & 9.8 & \\
\hline & $p, p^{\prime}$-DDT & $\begin{array}{l}\text { water, tap, reservoir } \\
\text { water }\end{array}$ & GC-ECD & 200 & $90.5-92.6$ & 9.6 & $\begin{array}{l}\text { Lambropoulou } \\
\text { et al. } 2007\end{array}$ \\
\hline & $p, p^{\prime}$-DDT & Water, wastewater & GC-MS & 101 & $50 \pm 10$ & 7.8 & Cortada et al. 2009 \\
\hline & $p, p^{\prime}$-DDE & $\begin{array}{l}\text { water, tap, reservoir } \\
\text { water }\end{array}$ & GC-ECD & 50 & $94.2-98.3$ & 5.4 & $\begin{array}{l}\text { Lambropoulou } \\
\text { et al. } 2007\end{array}$ \\
\hline & $p, p^{\prime}$-DDE & Water, wastewater & GC-MS & 25 & $43 \pm 5$ & 9.0 & Cortada et al. 2009 \\
\hline & $p, p$-DDD & Water, wastewater & GC-MS & 22 & $47 \pm 7$ & 6.9 & \\
\hline & $\alpha$-endosulfan & $\begin{array}{l}\text { water, tap, reservoir } \\
\text { water }\end{array}$ & GC-ECD & 200 & $83.3-90.4$ & 4.6 & $\begin{array}{l}\text { Lambropoulou } \\
\text { et al. } 2007\end{array}$ \\
\hline & & Water, wastewater & GC-MS & 64 & $47 \pm 6$ & 7.6 & Cortada et al. 2009a \\
\hline & $\beta$-endosulfan & Water, wastewater & GC-MS & 71 & $52 \pm 5$ & 7.9 & \\
\hline & $\alpha-\mathrm{HCH}$ & Water, wastewater & GC-MS & 87 & $103 \pm 8$ & 6.7 & \\
\hline
\end{tabular}




\begin{tabular}{|c|c|c|c|c|c|c|c|}
\hline $\begin{array}{l}\text { Sample } \\
\text { preparation }\end{array}$ & Compounds & Type of water & Analysis & $\begin{array}{l}\operatorname{LoD} \\
(\mathrm{ng} / \mathrm{l})\end{array}$ & $\begin{array}{l}\text { Recovery } \\
(\%)\end{array}$ & RSD (\%) & Ref. \\
\hline & $\beta-\mathrm{HCH}$ & Water, wastewater & GC-MS & 93 & $100 \pm 8$ & 6.5 & \\
\hline & $\gamma-\mathrm{HCH}$ & $\begin{array}{l}\text { water, tap, reservoir } \\
\text { water }\end{array}$ & GC-ECD & 20 & 97.2 & 3.2 & $\begin{array}{l}\text { Lambropoulou } \\
\text { et al. } 2007\end{array}$ \\
\hline & & Water, wastewater & GC-MS & 45 & $102 \pm 8$ & 6.5 & \\
\hline & $\delta$-HCH & Water, wastewater & GC-MS & 66 & $101 \pm 8$ & 8.2 & \\
\hline & PCBs & $\begin{array}{l}\text { Distilled water, river, } \\
\text { lake, tap water }\end{array}$ & GC-ECD & 2.5 & $88-102$ & $6.8-7.9$ & \\
\hline \multirow[t]{29}{*}{ HF-LPME } & aldrin & rainwater & $\begin{array}{l}\text { GC-MS } \\
\text { (SIM) }\end{array}$ & 59 & $79.3-98.7$ & 2.01 & $\begin{array}{l}\text { Lambropoulou } \\
\text { et al. } 2007\end{array}$ \\
\hline & & Water, seawater & $\begin{array}{l}\text { GC-MS } \\
\text { (SIM) }\end{array}$ & 6 & & 8.6 & \\
\hline & & seawater & $\begin{array}{l}\text { GC-MS } \\
\text { (SIM) }\end{array}$ & 59 & $83.6-89.5$ & 2.01 & \\
\hline & dieldrin & Water, seawater & GC-MS & 1 & & 5.7 & \\
\hline & & rainwater & GC-MS & 47 & $74.9-87.3$ & 2.32 & \\
\hline & & seawater & GC-MS & 47 & $91.4-97.1$ & 2.32 & \\
\hline & edrin & Water, seawater & GC-MS & 8 & & 4.7 & \\
\hline & & rainwater & GC-MS & 33 & $85.6-93.1$ & 1.93 & \\
\hline & & seawater & GC-MS & 31 & $89.3-90.0$ & 5.50 & \\
\hline & $p, p^{\prime}$-DDT & rainwater & GC-MS & 17 & $81.6-97.6$ & 1.66 & \\
\hline & & seawater & GC-MS & 17 & $81.7-94.7$ & 1.66 & \\
\hline & & Water, seawater & GC-MS & 1 & & 7.4 & \\
\hline & $p, p-\mathrm{DDE}$ & Water, seawater & GC-MS & 1 & & 10.6 & \\
\hline & $p, p^{\prime}$-DDD & rainwater & GC-MS & 28 & $85.0-108.4$ & 2.28 & \\
\hline & & seawater & GC-MS & 28 & $92.1-95.2$ & 2.28 & \\
\hline & & Water, seawater & GC-MS & 1 & & 7.4 & \\
\hline & endosulfan & rainwater & GC-MS & 28 & $79.4-90.1$ & 3.13 & \\
\hline & & seawater & GC-MS & 28 & $92.0-93.3$ & 3.13 & \\
\hline & $\alpha-\mathrm{HCH}$ & rainwater & GC-MS & 17 & $86.1-106.7$ & 13.72 & \\
\hline & & seawater & GC-MS & 17 & $91.8-93.6$ & 13.72 & \\
\hline & & water, seawater & GC-MS & 1 & & 6.6 & \\
\hline & $\beta-\mathrm{HCH}$ & rainwater & GC-MS & 29 & $87.4-111.6$ & 10.29 & \\
\hline & & seawater & GC-MS & 29 & $85.3-91.3$ & 10.29 & \\
\hline & & water, seawater & GC-MS & 5 & & 5.5 & \\
\hline & $\gamma-\mathrm{HCH}$ & rainwater & GC-MS & 13 & $93.4-112.6$ & 14 & \\
\hline & & seawater & GC-MS & 13 & $84.1-86.52$ & 14 & \\
\hline & & water, seawater & GC-MS & 3 & & 6.5 & \\
\hline & $\delta$-HCH & water, seawater & GC-MS & 2 & & 5.5 & \\
\hline & simazine & Deionized water & GC-MS & 10 & $94.3-104.5$ & $0.78-2.68$ & \\
\hline \multirow[t]{4}{*}{$\begin{array}{l}\text { DHT- } \\
\text { LPME } \\
\end{array}$} & dieldrin & Deionized, rainwater & GC-MS & 2 & 84.2 & 14.4 & Chen et al. 2009 \\
\hline & $o, p^{\prime}$-DDT & Deionized, rainwater & GC-MS & 4 & 94.4 & 6.5 & \\
\hline & $p, p-D D E$ & Deionized, rainwater & GC-MS & 4 & 99.6 & 10 & \\
\hline & $\alpha$-endosulfan & Deionized, rainwater & GC-MS & 5 & 85.8 & 12.2 & \\
\hline \multirow[t]{4}{*}{$\begin{array}{l}\text { DHS-TEH- } \\
\text { LPME }\end{array}$} & aldrin & Water, river water & GC-MS/MS & 0.33 & & 10.9 & Huang et al. 2009 \\
\hline & dieldrin & Water, river water & GC-MS/MS & 25 & $106 \pm 12$ & 16.3 & \\
\hline & $o, p-D D T$ & Water, river water & GC-MS/MS & 0.37 & $98 \pm 15$ & 17.4 & \\
\hline & $\alpha$-endosulfan & Water, river water & GC-MS/MS & 19 & $109 \pm 15$ & 12.5 & \\
\hline DLLME & aldrin & $\begin{array}{l}\text { Distilled, river tap, } \\
\text { surface water }\end{array}$ & GC-MS & 9 & $81-97$ & 7 & Chen et al. 2009 \\
\hline
\end{tabular}

Vyviurska, O. et al., Sample preparation procedures for analysis of organochlorinated pollutants... 


\begin{tabular}{|c|c|c|c|c|c|c|c|}
\hline $\begin{array}{l}\text { Sample } \\
\text { preparation }\end{array}$ & Compounds & Type of water & Analysis & $\begin{array}{l}\text { LoD } \\
(\mathbf{n g} / \mathbf{l})\end{array}$ & $\begin{array}{l}\text { Recovery } \\
(\%)\end{array}$ & RSD (\%) & Ref. \\
\hline & dieldrin & $\begin{array}{l}\text { Distilled, river tap, } \\
\text { surface water }\end{array}$ & GC-MS & 4 & $82-100$ & 9 & Cortada et al. 2009b \\
\hline & endrin & $\begin{array}{l}\text { Distilled, river tap, } \\
\text { surface water }\end{array}$ & GC-MS & 4 & $81-102$ & 8 & \\
\hline & $p, p$-DDT & $\begin{array}{l}\text { Distilled, river tap, } \\
\text { surface water }\end{array}$ & GC-MS & 4 & $75-93$ & 11 & \\
\hline & $p, p-D D E$ & $\begin{array}{l}\text { Distilled, river tap, } \\
\text { surface water }\end{array}$ & GC-MS & 2 & $81-99$ & 7 & \\
\hline & $p, p$-DDE & $\begin{array}{l}\text { Distilled, river tap, } \\
\text { surface water }\end{array}$ & GC-MS & 4 & $84-96$ & 8 & \\
\hline & $\alpha$-endosulfan & $\begin{array}{l}\text { Distilled, river tap, } \\
\text { surface water }\end{array}$ & GC-MS & 5 & $83-95$ & 6 & \\
\hline & $\beta$-endosulfan & $\begin{array}{l}\text { Distilled, river tap, } \\
\text { surface water }\end{array}$ & GC-MS & 25 & $85-103$ & 15 & \\
\hline & $\alpha-\mathrm{HCH}$ & $\begin{array}{l}\text { Distilled, river tap, } \\
\text { surface water }\end{array}$ & GC-MS & 3 & $101-113$ & 7 & \\
\hline & $\beta-\mathrm{HCH}$ & $\begin{array}{l}\text { Distilled, river tap, } \\
\text { surface water }\end{array}$ & GC-MS & 5 & $96-112$ & 25 & \\
\hline & $\gamma-\mathrm{HCH}$ & $\begin{array}{l}\text { Distilled, river tap, } \\
\text { surface water }\end{array}$ & GC-MS & 8 & $96-111$ & 5 & \\
\hline & PCBs & Well, river, seawater & GC-ECD & $1.0-1.5$ & $92-103$ & $4.1-11.0$ & Rezaei et al. 2008 \\
\hline \multirow[t]{25}{*}{ MASE } & alachlor & $\begin{array}{l}\text { Milli-Q, drinking } \\
\text { water }\end{array}$ & GC-MS & 10 & 72.3 & 10.7 & $\begin{array}{l}\text { Lambropoulou } \\
\text { et al. } 2007\end{array}$ \\
\hline & aldrin & Bidistilled water & GC-MS & 5 & & 10.9 & Hauser et al. 2004 \\
\hline & dieldrin & Bidistilled water & GC-MS & 2 & & 7.3 & \\
\hline & endrin & Bidistilled water & GC-MS & 2 & & 7.0 & \\
\hline & $p, p^{\prime}$-DDT & Bidistilled water & GC-MS & 2 & & 9.5 & \\
\hline & $o, p^{\prime}-D D T$ & Bidistilled water & GC-MS & 2 & & 7.9 & \\
\hline & $p, p^{\prime}-D D E$ & Bidistilled water & GC-MS & 2 & & 4.7 & \\
\hline & $p, p^{\prime}-D D D$ & Bidistilled water & GC-MS & 2 & & 6.8 & \\
\hline & $\beta$-endosulfan & Bidistilled water & GC-MS & 10 & & 7.4 & \\
\hline & $\alpha-\mathrm{HCH}$ & $\begin{array}{l}\text { Milli-Q, drinking } \\
\text { water }\end{array}$ & GC-MS & 0.01 & 81.8 & 15.5 & $\begin{array}{l}\text { Lambropoulou } \\
\text { et al. } 2007\end{array}$ \\
\hline & & $\begin{array}{l}\text { Deionized, river, } \\
\text { drinking water }\end{array}$ & GC-MS & $10-25$ & 107.6 & 5.2 & \\
\hline & & $\begin{array}{l}\text { Reagent water, } \\
\text { groundwater }\end{array}$ & GC-MS & 20 & 89 & 6 & Hauser et al. 2001 \\
\hline & & Bidistilled water & GC-MS & 5 & & 5.7 & Hauser et al. 2004 \\
\hline & $\beta-\mathrm{HCH}$ & $\begin{array}{l}\text { Milli-Q, drinking } \\
\text { water }\end{array}$ & GC-MS & 0.02 & 73.8 & 16.7 & $\begin{array}{l}\text { Lambropoulou } \\
\text { et al. } 2007\end{array}$ \\
\hline & & $\begin{array}{l}\text { Reagent water, } \\
\text { groundwater }\end{array}$ & GC-MS & 10 & & & Hauser et al. 2001 \\
\hline & & Bidistilled water & GC-MS & 10 & & 4.8 & Hauser et al. 2004 \\
\hline & $\gamma-\mathrm{HCH}$ & $\begin{array}{l}\text { Milli-Q, drinking } \\
\text { water }\end{array}$ & GC-MS & 0.04 & 98.5 & 16 & $\begin{array}{l}\text { Lambropoulou } \\
\text { et al. } 2007\end{array}$ \\
\hline & & $\begin{array}{l}\text { Reagent water, } \\
\text { groundwater }\end{array}$ & GC-MS & 10 & 93 & 9 & Hauser et al. 2001 \\
\hline & & Bidistilled water & GC-MS & 10 & & 6.0 & Hauser et al. 2004 \\
\hline & $\delta-\mathrm{HCH}$ & $\begin{array}{l}\text { Milli-Q, drinking } \\
\text { water }\end{array}$ & GC-MS & 0.01 & 68.7 & 17.1 & $\begin{array}{l}\text { Lambropoulou } \\
\text { et al. } 2007\end{array}$ \\
\hline & & $\begin{array}{l}\text { Reagent water, } \\
\text { groundwater }\end{array}$ & GC-MS & 25 & 105 & 8 & Yamini et al. 2008 \\
\hline & & Bidistilled water & GC-MS & 2 & & 5.4 & Hauser et al. 2004 \\
\hline & hexachlrobenzene & $\begin{array}{l}\text { Milli-Q, drinking } \\
\text { water }\end{array}$ & 0.02 & 47.3 & 2.6 & & $\begin{array}{l}\text { Lambropoulou } \\
\text { et al. } 2007\end{array}$ \\
\hline & & $\begin{array}{l}\text { Reagent water, } \\
\text { groundwater }\end{array}$ & 10 & 93 & 9 & & Yamini et al. 2008 \\
\hline & & Bidistilled water & 2 & & 6.0 & & Hauser et al. 2004 \\
\hline
\end{tabular}




\begin{tabular}{|c|c|c|c|c|c|c|c|}
\hline $\begin{array}{l}\text { Sample } \\
\text { preparation }\end{array}$ & Compounds & Type of water & Analysis & $\begin{array}{l}\text { LoD } \\
(\mathrm{ng} / 1)\end{array}$ & $\begin{array}{l}\text { Recovery } \\
(\%)\end{array}$ & RSD (\%) & Ref. \\
\hline & PCBs & Drinking water & & 10.0 & 72.3 & 10.7 & $\begin{array}{l}\text { Lambropoulou } \\
\text { et al. } 2007\end{array}$ \\
\hline \multirow[t]{14}{*}{$\begin{array}{l}\text { DLLME- } \\
\text { SFO }\end{array}$} & aldrin & $\begin{array}{l}\text { Deionized, tap, river, } \\
\text { agriculture water }\end{array}$ & GC-ECD & 7 & & 5.4 & Yamini et al. 2008 \\
\hline & & Tap, lake water & GC-ECD & 21.5 & $90.2-94.8$ & 8.5 & Leong et al. 2009 \\
\hline & dieldrin & $\begin{array}{l}\text { Deionized, tap, river, } \\
\text { agriculture water }\end{array}$ & GC-ECD & 19 & & 6.4 & Yamini et al. 2008 \\
\hline & endrin & $\begin{array}{l}\text { Deionized, tap, river, } \\
\text { agriculture water }\end{array}$ & GC-ECD & 14 & & 7.2 & \\
\hline & $p, p^{\prime}$-DDT & $\begin{array}{l}\text { Deionized, tap, river, } \\
\text { agriculture water }\end{array}$ & GC-ECD & 16 & & 5.5 & \\
\hline & $o, p^{\prime}$-DDT & Tap, lake water & GC-ECD & 25.1 & $85.1-90.0$ & 8.8 & Leong et al. 2009 \\
\hline & $p, p^{\prime}-\mathrm{DDE}$ & $\begin{array}{l}\text { Deionized, tap, river, } \\
\text { agriculture water }\end{array}$ & GC-ECD & 10 & & 6.3 & Yamini et al. 2008 \\
\hline & & Tap, lake water & GC-ECD & 28.3 & $86.3-102.5$ & 7.2 & Leong et al. 2009 \\
\hline & $p, p^{\prime}$-DDD & $\begin{array}{l}\text { Deionized, tap, river, } \\
\text { agriculture water }\end{array}$ & GC-ECD & 8 & & 4.9 & Yamini et al. 2008 \\
\hline & $\alpha$-endosulfan & $\begin{array}{l}\text { Deionized, tap, river, } \\
\text { agriculture water }\end{array}$ & GC-ECD & 16 & & 5.5 & \\
\hline & & Tap, lake water & GC-ECD & $12.1-19.7$ & $87.0-96.2$ & 7.6 & Leong et al. 2009 \\
\hline & $\beta$-endosulfan & $\begin{array}{l}\text { Deionized, tap, river, } \\
\text { agriculture water }\end{array}$ & GC-ECD & 9 & & 5.9 & Yamini et al. 2008 \\
\hline & & Tap, lake water & GC-ECD & 12.9 & $85.5-93.5$ & 5.8 & Leong et al. 2009 \\
\hline & $\gamma-\mathrm{HCH}$ & $\begin{array}{l}\text { Deionized, tap, river, } \\
\text { agriculture water }\end{array}$ & GC-ECD & 11 & & 5.8 & Yamini et al. 2008 \\
\hline \multirow[t]{4}{*}{ DLLE-LSC } & aldrin & River, reservoir water & GC-MS & $0.6-1.2$ & $93.9-104.5$ & $4.1-7.1$ & Tsai et al. 2009 \\
\hline & dieldrin & River, reservoir water & GC-MS & $0.4-1.1$ & $93.6-100.5$ & $8.2-8.9$ & \\
\hline & $\alpha$-endosulfan & River, reservoir water & GC-MS & $0.4-0.8$ & $99.5-102$ & $5.6-9.7$ & \\
\hline & $\beta$-endosulfan & River, reservoir water & GC-MS & $1.3-2.5$ & $93.1-101.6$ & $6.0-8.2$ & \\
\hline \multirow[t]{13}{*}{ USAEME } & aldrin & $\begin{array}{l}\text { Distilled, tap, well, } \\
\text { surface water }\end{array}$ & GC- $\mu \mathrm{ECD}$ & $2-6$ & $90-98$ & & Ozcan et al. 2009 \\
\hline & dieldrin & $\begin{array}{l}\text { Distilled, tap, well, } \\
\text { surface water }\end{array}$ & GC- $\mu \mathrm{ECD}$ & $2-16$ & $83-94$ & & \\
\hline & endrin & $\begin{array}{l}\text { Distilled, tap, well, } \\
\text { surface water }\end{array}$ & GC- $\mu \mathrm{ECD}$ & $2-16$ & $94-103$ & & \\
\hline & $p, p^{\prime}$-DDT & $\begin{array}{l}\text { Distilled, tap, well, } \\
\text { surface water }\end{array}$ & GC- $\mu \mathrm{ECD}$ & $2-16$ & $75-83$ & & \\
\hline & $p, p^{\prime}-D D E$ & $\begin{array}{l}\text { Distilled, tap, well, } \\
\text { surface water }\end{array}$ & GC- $\mu \mathrm{ECD}$ & $2-16$ & $98-100$ & & \\
\hline & $p, p$-DDD & $\begin{array}{l}\text { Distilled, tap, well, } \\
\text { surface water }\end{array}$ & GC- $\mu \mathrm{ECD}$ & $2-16$ & $95-100$ & & \\
\hline & $\alpha$-endosulfan & $\begin{array}{l}\text { Distilled, tap, well, } \\
\text { surface water }\end{array}$ & GC- $\mu \mathrm{ECD}$ & $2-16$ & $94-101$ & & \\
\hline & $\beta$-endosulfan & $\begin{array}{l}\text { Distilled, tap, well, } \\
\text { surface water }\end{array}$ & GC- $\mu \mathrm{ECD}$ & $2-16$ & $90-101$ & & \\
\hline & $\alpha-\mathrm{HCH}$ & $\begin{array}{l}\text { Distilled, tap, well, } \\
\text { surface water }\end{array}$ & GC- $\mu \mathrm{ECD}$ & $2-16$ & $100-103$ & & \\
\hline & $\beta-\mathrm{HCH}$ & $\begin{array}{l}\text { Distilled, tap, well, } \\
\text { surface water }\end{array}$ & GC- $\mu$ ECD & $2-16$ & $98-100$ & & \\
\hline & $\gamma-\mathrm{HCH}$ & $\begin{array}{l}\text { Distilled, tap, well, } \\
\text { surface water }\end{array}$ & GC- $\mu \mathrm{ECD}$ & $2-16$ & $100-103$ & & \\
\hline & & Ultrapure, tap water & GC-MS & 21 & $103-109$ & $6-9$ & Regueiro et al. 2008 \\
\hline & $\gamma-\mathrm{HCH}$ & $\begin{array}{l}\text { Distilled, tap, well, } \\
\text { surface water }\end{array}$ & GC- $\mu \mathrm{ECD}$ & $2-16$ & $100-102$ & & Ozcan et al. 2009 \\
\hline PC-HFME & PCBs & $\begin{array}{l}\text { An artificial sea water, } \\
\text { spiked tap water }\end{array}$ & GC-MS & $0.04-0.21$ & 83-93 & $1.6-8.6$ & Basheer et al. 2007 \\
\hline
\end{tabular}

Vyviurska, O. et al., Sample preparation procedures for analysis of organochlorinated pollutants... 


\section{Sample preparation for PAHs analysis}

Two ISO sample preparation procedures (ISO 17993:2002 and ISO 28540:2011) based on LLE with hexane are commonly used for PAHs analysis by GC and HPLC techniques. In both cases, one liter of water sample is dechlorinated with of sodium thiosulfate, and $\mathrm{pH}$ of water is adjusted to value 2 with sodium thiosulfate for HPLC analysis. Extract for GC analysis is cleaned though the cartridge filled with silica, and in the case of HPLC, extract is eluted from cleanup column including silica gel with mixture of dichloromethane/hexane (1:1). A number of alternative sample preparation procedures were developed for PAHs extraction with gas chromatography (Table 2). Multi-walled carbon nanotubes (MWCNTs) were used as a novel adsorbent for SPE procedure of 16 PAHs from environmental water samples (Ma et al. 2010). In this procedure, a $500 \mathrm{ml}$ water sample was loaded at the flow rate of $5.0 \mathrm{ml} / \mathrm{min}$, and after $30 \mathrm{~min}$ under vacuum (to remove residual water), analytes were eluted by $15 \mathrm{ml}$ of $n$-hexane at the flow rate of $1 \mathrm{ml} / \mathrm{min}$. Lower recoveries (about $10 \%$ ) were found for C18 sorbent material compared to MWCNTs. PAHs as nonpolar compounds can be easily adsorbed on MWCNTs than on C18, because the delocalized $\pi$-bond interaction between PAHs and MWCNTs is much stronger than the hydrophobic interaction between PAHs and C18 group. In the addition, low LoD was received in this case. Additionally, 24 PAHs were concentrated from wastewater by SBSE with a $10-\mathrm{mm}$ long stir bar coated with a $0.5 \mathrm{~mm}$ film thickness layer of PDMS. Extraction procedure was carried out from $50 \mathrm{ml}$ of non-filtered wastewater with $10 \%$ of methanol to prevent PAHs adsorption on the glassware during $4 \mathrm{~h}$ (Barco-Bonilla et al. 2011). Huertas et al. (Huertas et al. 2007) used two times longer stir bar for extraction of 7 PAHs in the presence of other priority substances from the European Water Framework Directive in seawater. However, in this case the increased amount of water sample $(100 \mathrm{ml})$ as well as a long extraction time $(12 \mathrm{~h})$ were required. Consequently LoD values of PAHs were decreased in one order of magnitude. SBSE and SPME procedures were compared by Popp et al. (Popp et al. 2003). One hundred micrometer PDMS SPME fibre or a $10 \mathrm{~mm}$ stir bar coated with a $0.5 \mathrm{~mm}$ layer of PDMS was used for $10 \mathrm{ml}$ of water sample during $1 \mathrm{~h}$. Since SBSE is characterized by higher phase ratio (volume of water phase to the volume of the PDMS phase), it results in higher recoveries and lower LoD values than SPME. The same fibre was tested for monitoring of degradation of PAHs after ultrasonic treatment from $5 \mathrm{ml}$ of spiked water sample (Psillakis et al. 2003), however the much worse quantification characteristics were received in this case. Another commercial fibre $(65-\mu \mathrm{m}$ PDMS/DVB) was used for concentration of 27 parent and alkylated PAHs with diverging polarities (Fernández-González et al. 2007). It was found that organic modified (e.g. methanol) which is usually recommended for prevention of PAHs adsorption on the glass walls, has negative influence on recoveries of PAHs. In addition, solution ionic strength and sample $\mathrm{pH}$ has shown little effect on the extraction of analytes. In general very low values of LoD comparable with best results of SBSE procedure was received. Alternative SPME fibre coating, such as electrochemically prepared dodecylsulfate-doped polypyrrole with a porous structure was applied for extraction of 8 PAHs (Mohammadi et al. 2005). Although $\mathrm{NaCl}$ was added to $10 \mathrm{ml}$ of water sample, LoDs were too high in comparison with other sample preparation techniques. Rezaee et al. (Rezaee et al. 2006) tested different combinations of an extraction solvent (carbon disulfide, carbon tetrachloride and tetrachloroethylene) and dispersive solvent (acetone, acetonitrile and methanol) for effective concentration of 16 PAHs by DLLME method. As the result, the higher extraction effectiveness was reached with tetrachloroethylene, whereas variations of recoveries for tested disperse solvents were not remarkable, but acetone is less toxic and cheaper. $8.0 \mu \mathrm{l}$ of tetrachloroethylene as extraction solvent and $1.00 \mathrm{ml}$ of acetone as disperser solvent were injected into $5.00 \mathrm{ml}$ of water solution and centrifuged for $1.5 \mathrm{~min}$. The increased volume of exctraction solvent decreased the enrichment factor and recovery. Cloudy state is not formed at a low volume of acetone, whereas at the high volume of acetone, the solubility of PAHs in water increases, therefore the extraction efficiency decreases. In comparison with LPME and SPME, DLLME was faster, simpler and inexpensive procedure with sufficient quantitative characteristics for PAH determination.

The SBSE and SPME sample preparation procedures were applied for HPLC analysis of PAHs (Table 3). The peak areas of the compounds in the SBSE chromatograms were 3- to 10-fold higher than those of the SPME chromatogram, and similarly LoD characteristics were better for SPME procedure (Popp et al. 2003). In-tube SPME device coated with PDMS was used for preconcentration of $4 \mathrm{PAHs}$ from $10 \mathrm{ml}$ of water sample (Bagheri et al. 2004). In this case nearly all the sample reacts with sorbent because of frequent withdrawing and ejecting the water sample from/into a vial. The received results were compatible with classical SPME procedure. Ultrasound-assisted surfactant-enhanced emulsification microextraction (UASEME) method was 
Tab. 2. Sample preparation procedures for PAHs before GC analysis.

\begin{tabular}{lllllll}
\hline $\begin{array}{l}\text { Sample } \\
\text { preparation }\end{array}$ & Type of water & Analysis & LoD (ng/L) & Recovery (\%) & RSD (\%) & Ref. \\
\hline SPE & Spiked real water & GC-MS & $2.0-8.5$ & $80.5-127$ & $1.2-12.1$ & Ma et al. 2010 \\
\hline SBSE & Wastewater & GC-MS & $2-10$ & $62-118$ & $2-26$ & Barco-Bonilla et al. 2011 \\
\hline & Seawater & GC-MS & $0.1-7.3$ & $90.2-97.3$ & $2.7-14.7$ & Huertas et al. 2007 \\
\hline SPME & Spiked ater & GC-MS & $0.05-0.5$ & $29-95$ & $2.5-21.7$ & Popp et al. 2003 \\
\hline & Water spiked & GC-MS & $0.2-1.2$ & $11-45$ & $3.0-16.8$ & \\
\hline & Spiked water & GC-MS & $10-700$ & & & Psillakis et al. 2003 \\
\hline & $\begin{array}{l}\text { Tap, well, superficial } \\
\text { water, seawater }\end{array}$ & GC-MS & $0.07-0.76$ & $59-103$ & $6.1-11.8$ & Fernández-González et al. 2007 \\
\hline DLLME & Sea water & GC-MS & $50-160$ & & $2.6-7.6$ & Mohammadi et al. 2005 \\
\hline
\end{tabular}

Tab. 3. Sample preparation procedures for PAHs before HPLC analysis.

\begin{tabular}{lllllll}
\hline $\begin{array}{l}\text { Sample } \\
\text { preparation }\end{array}$ & Type of water & Analysis & LoD (ng/L) & Recovery (\%) & RSD (\%) & Ref. \\
\hline SBSE & Spiked water & HPLC-MS & $0.3-2$ & & Popp et al. 2003 \\
\hline SPME & Water spiked & HPLC-MS & $1.0-10$ & & \\
\hline & Snow sample & HPLC-FLD & $1-6$ & & $2.6-6.3$ & Bagheri et al. 2004 \\
\hline UASEME & Tap, rain, waste water & HPLC-FLD & $0.6-62.5$ & $73-120$ & $1.8-10.8$ & Cheng et al. 2011 \\
\hline
\end{tabular}

applied for determination of PAHs in tap, river and waste waters (Cheng et al. 2011). It was found that the emulsification temperature and the equilibrium time have no significant effect on the extraction efficiency of the PAHs. 1-octanol, 1-dodecanol, $n$-tetradecane and cyclohexane were tested as extraction solvents, and the last one provides the best recoveries. It was found that nonpolar PAHs were more easily emulsified by the non-ionic surfactant (Tween 80 and Triton X10) than as ionic surfactant (SDS, CATB). At the result $20 \mu \mathrm{l}$ of cyclohexane and $10 \mu \mathrm{l}$ of Tween 80 were added to $5 \mathrm{ml}$ of aliquot of water containing $6 \%$ of NaCl. The LoDs of PAHs were in wide range $0.6-62.5 \mathrm{ng} / \mathrm{L}$.

\section{Acknowledgement}

This article was created with the support of the Ministry of Education, Science, Research and Sport of the Slovak Republic within the Research and Development Operational Programme for the project "University Science Park of STU Bratislava”, ITMS 26240220084, co-funded by the European Regional Development Fund.

\section{References}

Bagheri H, Salemi A (2004) Chromatographia 59: $501-505$.

Barco-Bonilla N, Romero-González R, Plaza-Bolaños P, Fernández-Moreno JL, Frenich AG, Martínez Vidal JL (2011) Analytica Chimica Acta 693: 62-71.

Basheer C, Vetrichelvan M, Valiyaveettil S, Lee HK (2007) Journal of Chromatography A 1139: 157-164.
Camino-Sánchez FJ, Zafra-Gómez A, Cantarero-Malagón S, Vílchez (2012) Talanta 89: 322-334.

Chen P-S, Huang S-P, Fuh M-R, Huang S-D (2009) Analytica Chimica Acta 647: 177-181.

Cheng J, Matsadiq G, Liu L, Zhou Y-W, Chen G (2011) Journal of Chromatography A 1218: 2476-2482.

Cortada C, Vidal L, Tejada S, Romo A, Canals A (2009a) Analytica Chimica Acta 638: 29-35.

Cortada C, Vidal L, Pastor R, Santiago N, Canals A (2009b) Analytica Chimica Acta 649: 218-221.

Cortazar E, Zuloaga O, Sanz J, Raposo JC, Etxebarria N, Fernández LA (2002) Journal of Chromatography A 978: 165-175.

Derouiche A, Driss M R., Morizur J-P, Taphanel M-H (2007) Journal of Chromatography A 1138: 231-243.

Fernández-González V, Concha-Graña E, MuniateguiLorenzo S, López-Mahía P, Prada-Rodríguez D (2007) Journal of Chromatography A 1176: 48-56.

Hauser B, Popp P (2001) Journal of Separation Science 24: 551-560.

Hauser B, Schellin M, Popp P (2004) Analytical Chemistry 76: 6029-6038.

Huang S-P, Chen P-S, Huang S-D (2009) Journal of Chromatography A 1216: 4347-4353.

Huertas C, Morillo J, Usero J, Gracia-Manarillo I (2007) Talanta 72: 1149-1156.

Lambropoulou DA, Albanis TA (2007) Journal of Biochemical and Biophysical Methods 70: 195-228.

Larsen BR (1995) Journal of High Resolution Chromatography 18: 141-151.

Leong M-I, Huang S-D (2009) Journal of Chromatography A 1216: 7645-7650.

Ma J, Xiao R, Li J, Yu J, Zhang Y, Chen L (2010) Journal of Chromatography A 1217: 5462-5469.

McFarland VA, Clarke JU (1989) Environmental Health Perspectives 81: 225-293. 
Mohammadi A, Yamini Y, Alizadeh N (2005) Journal of Chromatography A 1063: 1-8.

Ozcan S, Tor A, Emin Aydin M (2009) Water Research 43: 4269-4277.

Popp P, Bauer C, Hauser B, Keil P, Wennrich L (2003) Journal of Separation Science 26: 961-967.

Psillakis E, Ntelekos A, Mantzavinos D, Nikolopoulos E, Kalogerakis N (2003) Journal of Environmental Monitoring 5: 135-140.

Regueiro J, Llompart M, Garcia-Jares C, GarciaMonteagudo JC, Cela R (2008) Journal of Chromatography A 1190: 27-38.

Rezaee M, Assadi Y, Hosseini MRM, Aghaee E, Ahmadi F, Berijani S (2006) Journal of Chromatography A 1116: 1-9.
Rezaei F, Bidari A, Birjandi AP, Reza M, Hosseini M, Assadi Y (2008) Journal of Hazardous Materials 158: $621-627$

Tsai W-C, Huang S-D (2009) Journal of Chromatography A 1216: 5171-5175.

Yamini Y, Shariati S, Khalili-Zanjani MR, MansourBaghah S (2008) Analytica Chimica Acta 626: 166-173.

Yang F, Jin S, Meng D, Xu Y (2010) Chemosphere 81: 1000-1005. 